\title{
Inserción de la etnomatemática en la formación docente en la educación superior ecuatoriana
}

\author{
Juan Cadena Villota \\ Universidad Central del Ecuador \\ jrcadena@uce.edu.ec \\ Javier Collaguazo Singo \\ Universidad Central del Ecuador
}

Recibido: 9 - febrero-2018 / Aceptado: 31 - julio - 2018

\section{Resumen}

La etnomatemática como una nueva mirada de la matemática entraña en sus dimensiones: cognición, conceptualización, educación, epistemología, historia y política, lo cual implica un cambio en el paradigma educativo tradicional. Para este trabajo se consideran primordiales las instancias conceptuales e históricas, sin perjuicio de abordar las otras dimensiones. La educación matemática en el Ecuador ha venido asumiendo contenidos curriculares emanados solamente de la fuente euro centrista, no se ha considerado ni siquiera el análisis de los status espacio-temporales de la generación de los conceptos matemáticos, peor aún, no se han incorporado los saberes emanados de la raíz pre hispánica. El presente trabajo propone insertar la etnomatemática en la formación docente mediante contenidos con carga histórica crítica, realizando comparaciones, analogías y contrastes entre los diferentes lugares, ambientes y temporalidades del desarrollo matemático. Los contenidos se han escogido en base a diferentes lecturas y aproximaciones a nuestra realidad latinoamericana. Se presenta en forma de una propuesta metodológica con un análisis estadístico preliminar, conceptualizaciones contemporáneas y una propuesta de actividades didácticas, 
producto de dar respuesta a la necesidad de enfrentar el problema de la deficiente educación matemática a través de las consideraciones socioculturales, epistémicas y filosóficas inherentes a paradigmas concernientes a la etnomatemática.

Palabras clave: etnomatemática, praxis, teoría, didáctica, heurística.

\begin{abstract}
Ethnomathematics as a new look of mathematics involves its dimensions: cognition, conceptualization, education, epistemology, history and politics, which implies a change in the traditional educational paradigm. For this work, conceptual and historical instances are considered primordial, without prejudice to addressing the other dimensions. Mathematics Education in Ecuador has been assuming curricular contents emanating only from the Eurocentric source, not even the analysis of the spatio - temporal status of the generation of mathematical concepts has been considered, even worse, the knowledge has not been incorporated emanating from the pre-Hispanic root. The present work proposes to insert ethnomathematics in teacher training through critical historical content, making comparisons, analogies and contrasts between the different places, environments and temporalities of mathematical development. The contents have been chosen based on different readings and approaches to our Latin American reality. It is presented in the form of a methodological proposal with a preliminary statistical analysis, contemporary conceptualizations and a proposal of didactic activities, as a result of responding to the need to face the problem of poor mathematical education through the inherent sociocultural, epistemic and philosophical considerations to paradigms concerning ethnomathematics.
\end{abstract}

Keywords: ethnomathematics, praxis, theory, didactics, heuristics. 


\section{Introducción}

$\mathrm{E}$ n el Ecuador, las carreras de formación docente en matemática no han considerado en ningún caso, el estudio del desarrollo histórico de la matemática, lo que incide en un bajo rendimiento académico, una pobreza en la cultura científica y desconocimiento de la génesis de los conceptos matemáticos.

Uno de los problemas más acuciantes que encontramos los profesores de matemáticas es la búsqueda de problemas y situaciones didácticas que propongan un aprendizaje significativo en los estudiantes. La intervención didáctica es un proceso muy delicado, según Cantoral (2016), la construcción de sistemas conceptuales, más aún en proceso de elaboración, se articulan en los tres planos:

Primero, la problematización, como consecuencia de la naturaleza del saber matemático. En cualquier instancia, tanto el saber occidental, como el emanado de la fuente pre hispánica, se conecta con el segundo plano, muy importante para el conocimiento situado, es decir lo "local", lo cual implica una praxis social adyacente, las características del entorno, el contexto y el ideario. El tercer plano actuaría como el detonante de la búsqueda de una propia epistemología del conocimiento matemático, el conjunto de categorías que, provenientes de la actividad didáctica, cargadas de experiencias sistematizadas, produzcan paulatinamente una teoría del aprendizaje auténtico y autónomo.

La matemática aporta a la consecución de competencias que posibilitan resolver problemas fuera de ella, como potenciar sus capacidades de discernimiento, sistematización, creatividad, criticidad y toma de decisiones cruciales. Inherente a esta categorización de la matemática, la formación docente juega un papel muy importante. Debe existir una interrelación sostenible del nivel del conocimiento de la matemática y la carga pedagógica asociada mediante la transposición didáctica y además dicho conocimiento disciplinar debe ser complementado con una visión inclusoria del ambiente cultural que produzca un aprendizaje significativo (Blanco-Álvarez, Hilbert; Fernández - Oliveras, Alicia; Oliveras, María Luisa, 2017).

\section{Metodología}

Como punto de partida, veamos los resultados de una encuesta realizada en la Universidad Central del Ecuador, para aproximarnos a la realidad en la educación ecuatoriana.

\section{Ficha Técnica:}

Fecha de la encuesta: abril 2017.

Grupo de estudio: Estudiantes de Quinto Semestre de la Carrera de Formación Docente en Matemáticas

Estadísticos: Población: 82, Muestra: 50, Margen: 10\%, Nivel de confianza: 99\%

Propósito: mediante esta encuesta de acercamiento a la situación de enseñanza 
en la carrera de Formación Docente en Matemáticas, se pretende evidenciar a través de datos cuantitativos, la visión global de una problemática intrínseca a la educación matemática en el Ecuador, la ausencia de elementos curriculares o contenidos que propicien una formación en la cultura científica de la Historia de la Matemática como generadora de los conceptos en los planos cognitivos, procesales y aplicativos.

\section{Resumen holístico de la encuesta:}

Se ha separado las preguntas en varias categorías, a continuación, arrojamos algunos datos interesantes:

- Pregunta General: ¿Has recibido alguna asignatura sobre Historia de la Matemática en la Carrera?

Respuestas: NO en el 100\%. Lo cual evidencia que, hasta la actualidad la categoría histórica de la matemática está ausente de la formación académica. Dato por demás contundente.

- Preguntas sobre la génesis de los conocimientos matemáticos a través de la historia, en contexto cultural e inclusivo:

"Tu nivel de conocimiento acerca del nacimiento y desarrollo de los sistemas de numeración en el mundo es".

"¿Sabes algo sobre los 23 problemas del siglo XX planteados por David Hilbert en el Primer Congreso Mundial de Matemáticas en París de 1900?”.

"Tu nivel de conocimiento sobre las mujeres que han trascendido en la Historia de las Matemáticas es".

Análisis: Los resultados muestran un margen aproximado de $50 \%$ en conocimiento Nulo, $40 \%$ en Poco y $10 \%$ en conocimiento Suficiente.

Se da mayor peso de conocimiento nulo a la pregunta sobre los 23 problemas de Hilbert y sobre la presencia de las mujeres en la Historia de la Matemática, hecho que denota ausencia de nivel cultural y presencia evidente del modelo falocentrista occidental.

- Preguntas sobre teoría matemática básica en el contexto histórico y cientifico:

“¿Conoces los teoremas fundamentales de la Matemática y su aplicabilidad?"

“Conoces la historia del nacimiento del Cálculo Infinitesimal a través de la pugna Newton-Leibniz?”

“CConoces sobre la existencia de otras Geometrías diferentes a la Euclidiana?”.

Análisis: En los resultados vemos una aproximación del $40 \%$ en conocimiento Nulo. 50\% en Poco y 10\% en conocimiento Suficiente.

Resultados semejantes al bloque anterior, pero con la observación de mayor incidencia de conocimiento Nulo en los Teoremas Fundamentales y por sorpresa, ninguno respondió saber sobre las Geometrías No Euclidianas, lo cual es un indicio de la persistencia de la influencia de la Geometría greco-egipcia, y la evidencia del desconocimiento total de la Geometría Andina. 
- Preguntas sobre metodologías y enfoques de enseñanza

¿Estás de acuerdo que debemos saber sobre Historia de la Matemática para poder enseñarla?

La etnomatemática es una "nueva mirada sobre la matemática universal”. ¿Cuál es tu nivel de conocimiento de la etnomatemática?

Análisis: A la primera pregunta, el $98 \%$ contesta afirmativamente, lo cual indica que, de alguna manera, la historicidad de la matemática es atractiva a su perspectiva de formación. Al preguntarles sobre el status de conocimiento de la etnomatemática, el resultado es sorprendente, por así decirlo, el 82\% contesta Poco, el 16\% sabe Nada y el 2\% sabe lo Suficiente. En referencia al mayor porcentaje de Poco, se puede inferir que de alguna manera el término les es conocido, tal vez por los dos congresos internacionales realizados en la Universidad, lo cual no implica necesariamente que conozcan sobre ella.

Conclusiones breves: Como se puede inferir de los resultados, el nivel de conocimientos básicos en la génesis de los conceptos matemáticos, la historia, la etnomatemática, la inclusión y los paradigmas contemporáneos presenta un nivel deficiente en esta prueba estadística.

Es necesario pues, el abordaje de la incorporación de la historicidad matemática como componente básico en la formación docente, en la medida de su interpretación desde la crítica creadora, el análisis de coyuntura temporal y espacial y la revalidación de los conocimientos de las culturas ancestrales (Farías Gonçalves, 2016).

Por otro lado, el desarrollo de una práctica docente que involucre dos procesos independientes, pero no dicotómicos, la formación docente y la práctica en el aula, como una instancia de investigación de las teorías pertinentes de aprendizaje y enseñanza, su aplicación en la praxis cotidiana y la necesaria evaluación integral es imperante.

Se tomarán en cuenta varias aristas de estudio en la formación de los maestros, tomando en cuenta el enfoque de la inserción de la etnomatemática como un proceso que viabilice la extrapolación de las prácticas hacia la construcción de un constructo teórico, evidentemente perfectible a través, precisamente de la relación bidireccional dialéctica de la praxis y la teoría.

Entonces, en la medida de la problemática planteada, sugerimos una propuesta que disminuya el fracaso escolar en el aprendizaje de la matemática a partir de un programa de formación docente, que tiene como núcleo central la etnomatemática, como una nueva mirada en la educación, en lo antropológico, lo histórico y lo educativo (D’Ambrosio U. , 1990). Tomando en cuenta que, los conocimientos matemáticos acumulados a través de las generaciones se sustentan, entre otras, en ideas concernientes a la comparación, clasificación, medida, operatividad y generalización, éstos deben ser explicados bajo las características fenomenológicas de un 
contexto histórico, cultural, social, político e ideario (Rosa \& Orey, 2016). Debemos considerar además que la mirada de la etnomatemática concibe una episteme distinta a la occidental euro centrista, con un ethos que mide la dimensionalidad del tiempo y el espacio en contextos contrastantes con la perspectiva de la matemática proveniente del flujo europeo, árabe, babilonio y egipcio. Se hace necesaria entonces generar una corriente para recobrar, recrear y reinventar los aportes de los pueblos aborígenes americanos. No puede estudiarse un concepto o idea matemática sin mirar su contenido en la línea del tiempo, y más allá del nicho histórico, en las condiciones de la sociedad, en su realidad como constructo ontológico y su relación con la naturaleza (Cadena $\&$ Trujillo, 2016).

El proceso de enseńanza-aprendizaje de la matemática en Ecuador se ha basado principalmente en la aceptación y reproducción acrítica del modelo de racionalidad occidental. De su parte la racionalidad andina reconoce la alteridad (diferencia), como algo esencial que admiten y se enriquecen entre otras, con la razón del mito y con otras formas de sensibilidad en la comprensión del mundo, la simetría (reciprocidad, que se refleja en una visión dualista de la realidad) y la no arbitrariedad (complementariedad) (Esterman, 2015).

En este contexto, el hombre andino construye en relación al ser humano una identidad colectiva (Lizcano, 2006). Dentro del enfoque global, se asume que el objetivo será: conocer las concepciones de la cultura y la educación contemporáneas en un diálogo con la antropología, la historia, la sociología y psicología educativa, procurando explicar la manera en la que tales corrientes confluyen en la pedagogía de la matemática. En el enfoque local, se toman en cuenta los aspectos referentes a las características propias de cada sector cultural, tomando en cuenta las condiciones propias de la generación de la cognición y conceptualización de los elementos matemáticos, como el conteo, la medida, la clasificación y la generalización (Rosa \& Orey, 2016).

Tomando en cuenta que la noción de "cultura" es difícil de demarcar y aplicar en el campo de la etnomatemática, porque implica contextos históricos, políticos e incluso significados diferentes en cuanto al idioma, los tópicos a tratar deberán tener características que permitan un modelo epistemológico que conjugue lo universal con lo particular ( Kragh Sørensen, 2014).

Es importante también reconocer las diferentes concepciones éticas de las diferentes culturas y propender hacia un ethos universal (Cadena \& Trujillo, 2016).

Los tópicos para ser tomados en cuenta en la praxis deberán partir de las fuentes que se emanan de la etnomatemática como constructo de una nueva mirada de las matemáticas, es decir desde la visión antropológica, histórica y educativa (D’Ambrosio U. , 1990).

Se debe señalar que el Proyecto de Investigación Etnomatemáticas Runayupay, de la Universidad Central del Ecuador, propuso a las instancias directivas de la carrera de Formación Docente en Matemáticas de la Universidad Central, la incorporación 
de la cátedra Etnomatemática en la malla curricular del Re - Diseño de la Carrera, lográndose que se incorpore para el año 2019. Para lo cual se aprobó el contenido de la asignatura en cuatro unidades:

UNIDAD I. Introducción a la historia de las ciencias, la historia de la matemática en Europa, América, Asia y África.

UNIDAD II. Aritmética, astronomía, álgebra y arte en Europa, América, Asia y África.

UNIDAD III. Los sistemas de referencia, el cálculo y la noción del infinito.

UNIDAD IV. Desde el posmodernismo hasta nuestros días.

Por lo tanto, en este artículo no podemos analizar sobre los resultados de la inserción de la etnomatemática en la formación docente, ya que no está vigente aún.

En este sentido, el presente trabajo presenta una recopilación de reflexiones y actividades emanadas de la práctica en aulas de la carrera de Formación Docente en Matemáticas, específicamente en la cátedra de Didáctica de las Matemáticas, Tercero y Cuarto Semestre, en un período entre el 2015 y 2017.

Estas actividades también han sido expuestas en cursos de actualización docente, concretamente con los profesores de matemáticas de los colegios del Distrito Metropolitano de la ciudad de Quito, alcanzando a un conglomerado de alrededor de 200 profesores en ejercicio de la docencia.

Con seguridad, estas consideraciones y otras muchas más que provengan de la praxis y la actualización permanente en vertientes que aporten a formular estrategias metodológicas contemporáneas nutrirán la futura cátedra de Etnomatemática en su puesta en práctica en 2019.

Su estructura parte del siguiente esquema: Práctica docente y construcción teórica de modelos específicos de aprendizaje, tomando en cuenta actividades pertinentes a ello. No se pretende el convertirse en un manual per se de actividades aplicadas a un solo tema, sino más bien una propuesta adaptable a los requerimientos del lector, con la libertad de ajustarlos a su propia experiencia en el aula.

Comencemos por el diseño de actividades, un proceso mediante el cual se preparan de acuerdo a las experiencias dinámicas en el aula, nos referimos a una "mirada profesional" de las actividades, mediante la cual identificamos destrezas en el sentido de focalizar los aspectos relevantes de la situación de enseñanza, utilizando el contexto específico para analizar los momentos didácticos que aparecen en el aula y realizar las respectivas conexiones con teorías matemáticas y pedagógicas (Llinares, 2012).

Vamos a considerar algunas actividades de enseñanza de la matemática en las cuales intervienen diversas facetas, tomando en cuenta la génesis de ciertos conceptos, el contexto histórico y aún la incorporación de nuevas tecnologías, pero, en primer lugar: esa ineludible confianza en el hecho simple de que la capacidad de los aprendices es la generadora del conocimiento. 
Su redacción, en algunas partes, permite la licencia literaria de hablar en primera persona, por la intencionalidad de que el lector asuma la actividad de manera más vinculada a su propia asimilación y percepción.

\section{Actividades propuestas para la inserción de la Etnomatemática}

\section{Descubrir un número que relaciona proporciones}

Descripción:

Partiendo del hecho didáctico de relacionar el aprendizaje con las experiencias y manipulación de elementos que conduzcan a interiorizar la aprehensión por medio del discernimiento, la actitud crítica y una coordinación dialéctica entre los saberes previos y los saberes por aprender, esta guía se desarrollará con el objetivo de descubrir ciertas propiedades de las proporciones. Para ello se realizarán algunas actividades que tienen que ver con la interpretación cuantitativa de los fenómenos naturales. El objetivo es el descubrir relaciones que se repiten en procesos y fenómenos y comprobar estas relaciones por medio de algoritmos algebraicos y aritméticos.

Fundamentación teórica: El encontrar "un número especial" en forma heurística tiene dos perspectivas: desde un punto de vista geométrico y algebraico.

Vamos a dividir un segmento en dos partes, a la primera le denotaremos con $\mathbf{x} y$ la segunda es un segmento con valor $\mathbf{1}$, de tal manera que: al dividir la parte mayor para la parte menor, nos dé el mismo resultado que al dividir la parte mayor para todo el segmento de donde se deduce la siguiente ecuación cuadrática: $\boldsymbol{x}^{2}-\boldsymbol{x}-\mathbf{1}=\mathbf{0}$. La solución positiva de esta ecuación es el número que buscamos.

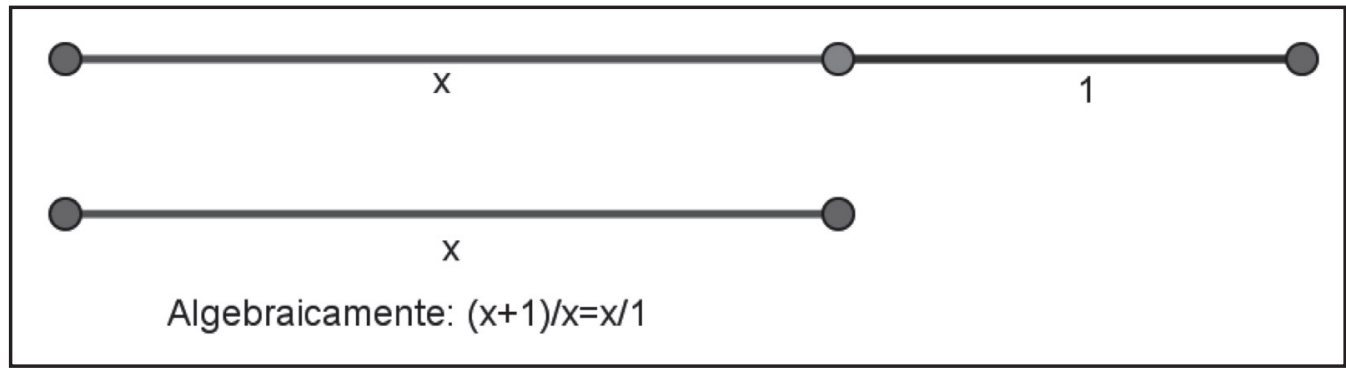

Gráfico 1. Fuente: elaboración propia

El matemático italiano Leonardo de Pisa, más conocido como Fibonacci (11701250), fue quien logró introducir la numeración árabe-hindú en Europa. A Fibonacci también se le atribuye la famosa sucesión que lleva su nombre:

Esta sucesión tiene muchas aplicaciones en la vida real. Pero hay una propiedad que nos interesa mucho: si dividimos cada número para su anterior a partir del tercer elemento, es decir: 
Tomemos unos más grandes:

Como puedes observar, mientras más grandes sean los números, la división entre dos elementos consecutivos (el más grande para el más pequeño) de la sucesión de Fibonacci, se acerca al misterioso número que buscamos.

Veamos ahora algunas curiosas apariciones de este número en la naturaleza, asombrosamente, este número aparece en diferentes facetas de la vida real:

En las ramas de los árboles, en los girasoles, en las espirales de los caracoles, en las telarańas, en las galaxias, en las tarjetas de crédito, en los copos de nieve. En manifestaciones artísticas como las pinturas de Leonardo Da Vinci, de Dalí, de Velásquez, Miguel de Santiago (pintor quiteño), etc.

Esta introducción teórica ha sido deliberadamente sintética, con el fin de prepararte para que tú mismo descubras este número mágico, oculto por ahí, de tal manera que, cuando lo encuentres entiendas que la naturaleza utiliza un lenguaje para comunicarse con nosotros y ese lenguaje lo podemos descifrar gracias a ...por supuesto, gracias a la matemática.

Recursos y materiales: El principal recurso es tu imaginación y tu disposición a descubrir conocimientos nuevos, lo demás viene por añadidura.

Primero, vamos a dividir la clase en grupos de 4 estudiantes cada uno. Se hará en forma aleatoria. Importante: Cada grupo se pondrá un nombre de combate (digamos científico), como Fibonacci, Phi, Gauss, Galois, Inca en la ciencia, Cañari, Pasto, etc.

Se nombrará un capitán de grupo, quien se encargará de la organización, disposición de tareas, presentaciones, etc.

Actividades de cálculo e investigación:

Cada grupo responderá las siguientes preguntas por separado:

A ese número (raíz positiva) se le llama con la letra griega:

Se le puso esta letra en honor a:

Este número pertenece al conjunto de los números:

¿Qué es la divina proporción? (explica brevemente)

¿Por qué se le llamará a este número: el número áureo?

¿Qué es la sección áurea?

¿Quién mismo era el tal Fibonacci? (una biografía corta)

¿Cuál es la mecánica para encontrar los números de la sucesión de Fibonacci? Encuentra los 12 primeros términos (manualmente, sin calculadora)

¿Puedes encontrar el término 100 de la sucesión de Fibonacci?

Actividades de aplicación:

Cada grupo escogerá un elemento concreto de cada uno de las siguientes categorías para explicar la aparición de este número en la vida cotidiana.

En la naturaleza (frutas, flores, árboles, animales, etc.)

En el arte (pinturas, esculturas, dibujos, etc.)

En la vida moderna (tarjetas de crédito, billetes, carnés, etc.)

Cada grupo presentará su ponencia en el aula. Disponen de 15 minutos para 
hacerlo. Cada grupo utilizará la herramienta que juzgue conveniente para la exposición. Habrá preguntas del resto de la clase

Actividades de comunicación:

Para culminar con nuestro proceso de búsqueda e información, es necesario que compartamos los conocimientos adquiridos. Para lo cual se abrirá un foro, que está en la plataforma virtual del curso, en el que debes participar independientemente de tu grupo. Es decir, esta es una tarea individual.

La consigna principal del foro es: ¿ڤHay matemáticas en la naturaleza?

De esta consigna se desprenden los siguientes hilos conductores:

¿Para qué sirven las matemáticas?, ¿Por qué las matemáticas se hacen difíciles?, ¿Los animales utilizan las matemáticas?

Ahora, desde la perspectiva de la etnomatemática, abordaremos el mismo problema, distante en tiempo y distancia, cultura y filosofía, sin embargo, conservando su esencia: leer a la naturaleza a través de la matemática. En este caso, la geometría: en el Sol de los Pastos podemos observar que se puede inferir la serie de Fibonacci, refiriéndonos al interesante trabajo de Víctor Samuel Albis (Albis, 1987).

En cuanto a la evaluación de la actividad, se propone utilizar rúbricas sencillas de aproximación a la cuantificación, pero con carácter cualitativo.

\section{Actividades basadas en el esquema CDR}

Font (2007), citado por (Planas \& Alsina, 2009), afirma que este esquema propone la utilización de un sistema dinámico al enfocar un tema concreto como actividad didáctica. Su significación: C: Contexto. D: Des contexto. R: Re contexto.

Veamos un ejemplo: Introducción al Cálculo Diferencial. Este tema se propone por ser una instancia poco comprendida en el proceso de enseñanza de la matemática, su propósito es generar conceptos afines en un entorno agradable que contribuyan a la comprensión del tema, que de por sí es un poco complicado.

Objetivos:

- Comprender el concepto de derivada, mediante la utilización de una aplicación informática que provoque en el alumno una ruptura con la forma de la enseñanza formal de la matemática.

- Asimilar la derivada y tratarla en su verdadero significado: medida del cambio.

- Contextualizar esta noción de cambio en un ambiente de génesis histórica y proyectarla a nuestro entorno social.

Idea base:

Se quiere conseguir el objetivo claro de comprender el concepto de derivada con la incorporación de varios elementos: asociación con la idea de cambio, contribución histórica, acercamiento al concepto matemático (sin demasiada formalidad), aplicación matemática y utilitaria.

Se contextualizará el sentido de la derivada en la parte histórica, el proceso de 
adjudicación de su creación cuyos protagonistas son Newton y Leibniz, su significación en cuanto a la tarea de acercarse al concepto de infinito.

Se des contextualizará el tema a través de unos conceptos matemáticos que, por una parte, formalicen el concepto de derivada y por otro lado sean operacionales para entender el fenómeno.

Se re contextualizará nuevamente el tema para acercarse a las aplicaciones de la derivada en un ambiente de operatividad funcional, tomando en cuenta la derivada como razón de cambio, en un escenario global, la derivada se conectará a través de contextos de la vida cotidiana, la física, la economía, la ecología, etc.

A través de la mirada etnomatemática, se re contextualizará en fenomenologías como la tasa de crecimiento de la migración del campo a las ciudades, el aumento del consumismo en las urbes, la rapidez de la pérdida de ciertas características inherentes a las culturas ancestrales, la etnolingüística, etc.

Otro ejemplo:

Consideremos que en geometría básica se está tratando el Teorema de Pitágoras. Aplicando nuevamente el esquema CDR, la contextualización se efectuará evocando la historia de la Escuela Pitagórica en la Isla de Samos y su huida a Crotona, la controversia entre la concepción metafísica de la cuantificación de la naturaleza.

Un marco de referencia histórico y filosófico es propicio para entender la fenomenología del Teorema y su trascendencia. Incluso las referencias a las culturas de Mesopotamia y la demostración china, son importantes para provocar en el alumno el interés en el tema.

En la des contextualización, se vuelve al aparato matemático con construcciones simples, pero sistemáticas, construir los cuadrados en los catetos y demostrar que la suma de sus superficies encaja en el área del cuadrado construido en la hipotenusa. Es necesario señalar que la construcción geométrica desemboca a la interpretación algebraica y analítica.

Se re contextualizará, en este caso, con una propuesta que remueva la concepción clásica del Teorema, por ejemplo, construyendo semicírculos en los lados del triángulo y pidiendo a los estudiantes que verifiquen si se cumple con la hipótesis pitagórica.

En nuestro enfoque local, la chakana andina nos sirve perfectamente para la verificación del Teorema de Pitágoras, proponiendo por ejemplo la construcción de triángulos rectángulos y comprobando el Teorema en el GeoGebra. Además, la chakana es un elemento identitario andino que encierra una semiótica propia, en cuanto a sus simetrías, ángulos, biparticiones, triparticiones y tetra particiones, es entonces un medio ideal para estudiar parte de la geometría plana con una gran carga de acercamiento cultural, histórico y didáctico. 


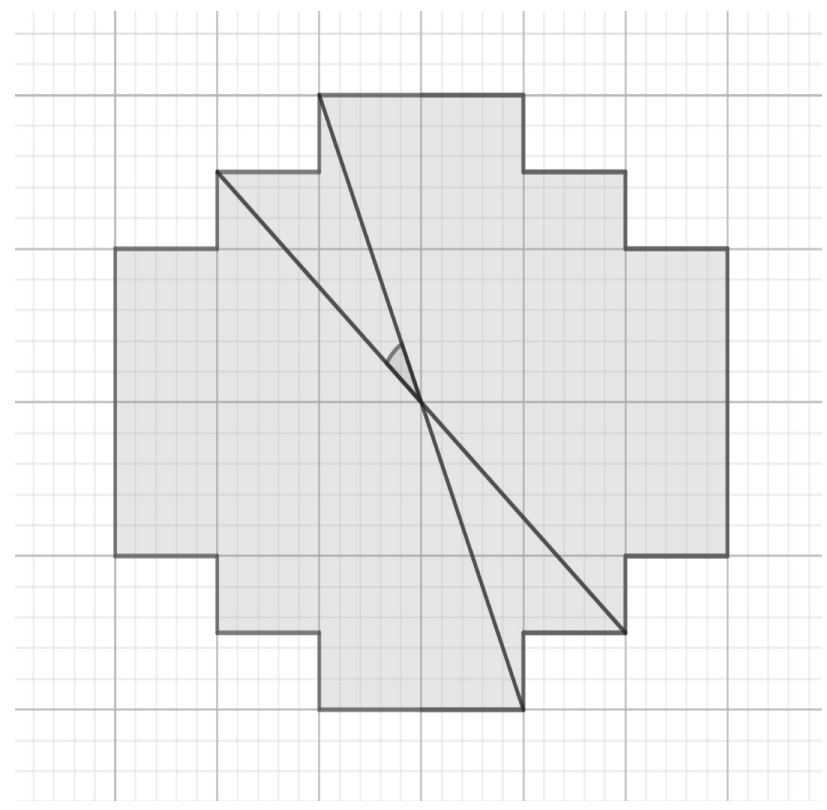

Gráfico 2. Chakana andina. Fuente: elaboración propia.

\section{Una recreación del Camino del Inca}

Nos referiremos a una concepción andina muy importante, entre muchas otras, la palabra "Pacha", acepción equivalente al "Cosmos" griego, sin embargo, en el mundo andino, esta palabra denota tanto "espacio", como "tiempo", coincidencia o no con la Teoría Especial de la Relatividad. Esto deriva, entre tantas disquisiciones, el planteamiento de la "Pachasofía", con sus complejidades epistemológicas y filosóficas (Sobrevilla, 2008).

Es interesante también la interpretación de la famosa frase del Racionalismo francés: "cogito ergo sum", a través de la llamada Pachasofía Andina: "celebramus ergo sumus" (Esterman, 2015). Donde lo cartesiano se trastoca de categoría antropocéntrica, individualista y segmentaria en una visión del mito, la celebración, la integración y lo comunitario.

Pues bien, la actividad, ubicada en este marco es una clase de matemáticas en cualquier centro escolar de nuestra región:

Contexto: Digamos que Inti (Sol en quechua) y Quilla (Luna en quechua), son dos chasquis, ellos son emisarios de Huayna Cápac, que recorren el Camino del Inca (Qhapaq Nan), a decir de Guamán Poma de Ayala, "un caracol que partía de Colombia llegaba vivo al Cuzco".

Inti y Quilla están entrenando para su larga caminata y deciden hacer una carrera de prueba de 100 metros.

Quilla es muy rápida y en la primera carrera le gana a Inti con 5 metros de distancia, es decir llega a la meta de los 100 metros en el instante en que Inti completa 95. Esta 
muchacha además es muy justa y le propone a Inti repetir la carrera una segunda vez, en esta ocasión, le dice a su compañero: "como yo te aventajé con 5 metros en la primera carrera, ahora voy a salir 5 metros antes de la línea de partida, para equilibrar la competencia, ¿qué te parece?", Inti acepta gustoso y corren otra vez.

Bajo esas premisas es lógico preguntar: ¿Si en la segunda carrera corren con las mismas velocidades que tuvieron en la primera, ¿quién ganará esta vez?

Entonces nos ubicamos en el descontexto: Los estudiantes se ponen a reflexionar en el problema. José levanta la mano luego de meditar un instante y le dice al profesor: "es lógico que: si en la primera carrera Quilla le aventajó a Inti con 5 metros, en la segunda carrera, al "devolverle" esa ventaja, lo más natural es que lleguen iguales".

El maestro pide opiniones, ¿está correcto el razonamiento de José?, la mayoría de la clase asiente, ¡claro, es lógico!

Sin embargo, María, caracterizada por su actitud siempre crítica y cuestionadora se atreve a opinar: “...pero, si Quilla ganó en la primera carrera, es lógico que corrió con mayor velocidad que Inti, es decir utilizó menor tiempo para recorrer la misma distancia. En la segunda carrera, Quilla recorrerá 5 metros más que Inti, pero es más veloz que él. En todo caso, vale preguntarse si hace menor o mayor tiempo que Inti en la segunda carrera".

El maestro sonríe y propone seguir el razonamiento de María. Les pide a los alumnos reflexionar sobre la relación entre las dos velocidades, que son constantes en las dos carreras: Esta proporción se mantiene constante. ¿Cómo la podremos utilizar para comparar los tiempos en la segunda carrera?, si sabemos que, en ésta, las distancias están en una relación de .

Se recontextualizará, luego de que los alumnos resuelvan el problema, al pedirles que hagan una interpretación geométrica del problema a través de construcción de segmentos y su proporcionalidad.

También se puede proponer la realización del problema en condiciones reales, a forma de experimento. Es decir, en este caso también utilizamos la incorporación de un etnomodelaje (Bassanezi, 2002), como una estrategia didáctica que relacione el conocimiento matemático teórico con las vivencias del aprendiz, en un contexto de aprendizaje significativo

Utilizamos seguidamente la perspectiva de "mirar con sentido" a las actividades realizadas por los estudiantes (Llinares, 2012).

\section{Tareas para el maestro:}

Tu tarea es analizar las respuestas de José y de María: ¿qué implicaciones deductivas, algorítmicas e intuitivas están en su razonamiento? María rompe la visión intuitiva del problema, ¿de qué manera se puede utilizar esta situación en el momento didáctico?

En esta actividad denotamos el manejo conceptual de las proporciones aritméticas y geométricas desde un punto de partida histórico e identitario, con- 
tribuyendo así a la construcción de una base epistémica local en armonía con actividades concretas.

De la misma manera, se podrán construir actividades didácticas que permitan desarrollar competencias docentes, basadas en la reflexión crítica, en la autonomía y la búsqueda del conocimiento matemático, en detonante de la creatividad y el compromiso por contribuir a la sociedad con una educación matemática de calidad, integral en el sentido ontológico, axiológico y epistemológico.

Es importante subrayar en el diseño de las actividades los elementos sustanciales que valoran el aporte a la construcción del conocimiento y su ulterior transposición a las aulas de secundaria. La comunicación es fundamental en base a la mayéutica socrática, los paradigmas educativos contemporáneos, el uso de herramientas informáticas, etc.

\section{A manera de conclusión}

El presente trabajo es fruto de reflexiones, investigación y actividades desarrolladas in situ. ${ }^{1}$ La construcción de una episteme propia en matemáticas es un camino en construcción, muy arduo y seguramente con muchos escollos. Pero estamos convencidos que dicha construcción es posible, citando a Ángel Ruiz: “...Se trata de medir con precisión el tejido de la realidad con sus puntos fuertes y vulnerabilidades y aportar un currículo capaz de hacer progresar la Educación Matemática..." (Ruiz, 2014).

En definitiva, veamos algunos elementos importantes en la contribución de la praxis a la teoría:

- El desarrollo de competencias en el docente que le permitan desarrollar investigación educativa en el campo de la educación matemática.

- La práctica de "mirar con sentido" a las actividades sistemáticas planteadas en clase, es decir el análisis, la retroalimentación y el constante monitoreo de los resultados alcanzados en el aula, que se constituye en el verdadero laboratorio de pragmatismo didáctico.

- La incorporación de categorías fundamentales en la enseñanza de la matemática, esto es la Historia de la Matemática, como punto de partida, análisis de coyunturas genealógicas del saber matemático, la construcción de los conceptos generados desde lo intuitivo hacia lo analítico.

- La consideración de la etnomatemática como una "nueva mirada" de la Educación Matemática, con todos sus matices intrínsecos, la globalidad, la localidad, la dialéctica y dialoguicidad entre diferentes culturas en el tiempo y el espacio.

1 Actividades realizadas en dos contextos: como parte de la cátedra de Didáctica de la Matemática en la carrera de Formación Docente y como exposición en cursos de actualización docente de maestros ecuatorianos, conferencias congresos y talleres. 
- Por último, consideramos que los trabajos de quienes realizamos educación matemática no pueden cercarse a fronteras imaginarias, impuestas por el mismo colonialismo, es necesario el establecer el norte de todo intento por lograr alcanzar un episteme andino, nuestro, posible, urgente. Solo necesitamos trabajar juntos.

\section{Referencias}

Albis, V. S. (1987). Las proporciones del Sol de los Pastos. Boletín de Matemáticas, Vol. XXI (2-3), 110-134.

Bassanezi, R. (2002). Ensino-aprendizagem com modelagem matemática. [Teaching-learning with mathematical modelling]. São Paulo: Editora Contexto.

Blanco-Álvarez, Hilbert; Fernández - Oliveras, Alicia; Oliveras, María Luisa. (agosto de 2017). Formación de profesores de matemáticas desde la etnomatemática: estado de desarrollo. Bolema, Boletim de Educação Matemática, v. 31(n. 58), 564-589.

Cadena, J., \& Trujillo, J. (2016). El pensamiento mito-lógico como sistema cognitivo de las etnociencias. Anales, 43-74.

Cantoral, R. (2016). Teoría socioepistemológica de la matemática educativa. Estudios sobre la construcción social del conocimiento. México: Gedisa.

D'Ambrosio, U. (1990). Ethnomathematics: The Art or Technique of Explaining or Knowing. Sao Paulo: Ática.

Esterman, J. (2015). Filosofía andina. Quito: Ediciones Abya-Yala.

Farías Gonçalves, P. G. (2016). Etnomatemática: Fragmentos históricos, fundamentos e perspectivas. Curitiba: CRV.

Font, V. (2007). Comprensión y contexto: Una mirada desde la didáctica de las matemáticas. Barcelona.

Kragh Sørensen, H. (2014). The End of Proof? The Integration of Different Mathematical Cultures. Mathematical Cultures, 141-143.

Lizcano, E. (2006). Metáforas que nos piensan sobre ciencia, democracia y otras poderosas ficciones. Barcelona: SKP.

Llinares, S. (2012). Formación de profesores de matemáticas. Caracterización y desarrollo de competencias docentes. Cuadernos de Investigación y Formación en Educación Matemática. Año 7. Número 10. pp 53-62. Costa Rica, pp 53-62.

Planas, N., \& Alsina, A. (2009). Buenas prácticas en la enseñanza de las matemáticas. Educación Matemática y Buenas Prácticas, 9-29.

Rosa, M., \& Orey, C. (2016). State of the Art in Ethnomathematics. ICME-13 Topical Surveys. Current and Future Perspectives of Ethnomathematics as a Program, 12-14.

Ruiz, Á. (2014). Compromisos y acciones para una reforma fundamentada del currículo. Perspectiva de la praxis en educación matemática. Universidad de Costa Rica, Costa Rica, 12-14. 
Sobrevilla, D. (2008). La filosofía andina del P. Josef Estermann. Solar, n. o 4, año 4, Lima, pp. 231-247. 